\title{
Incidence of hydronephrosis in severe uterovaginal or vault prolapse
}

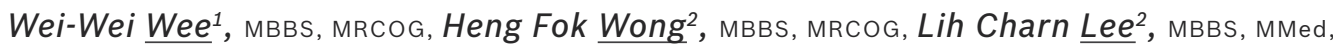
How Chuan $\underline{\mathrm{Han}}^{2}$, MBBS, MRACOG

INTRODUCTION We aimed to evaluate the local incidences of hydronephrosis and renal impairment in the presence of severe uterovaginal or vault prolapse, and determine whether treatment by surgery or ring pessary resulted in the resolution of hydronephrosis in these patients.

METHODS This was a retrospective case study of 121 patients who presented with severe uterovaginal or vault prolapse. All patients who had fourth degree uterovaginal or vault prolapse, and underwent renal ultrasonography and renal function blood tests were included in the study. Follow-up imaging for hydronephrosis was performed to determine the outcome after patients received treatment.

RESULTS The mean age of the study population was 66.1 years. The overall incidence of hydronephrosis was $20.6 \%$. The incidence of hydronephrosis in patients with severe vault prolapse was $7.1 \%$, while that in patients with severe uterovaginal prolapse was $22.4 \%$. Of the 25 patients with hydronephrosis, $16(64.0 \%)$ had complete resolution of hydronephrosis after treatment, $5(20.0 \%)$ had residual but smaller degrees of hydronephrosis, and 4 (16.0\%) were lost to follow-up. The incidence of renal impairment was 3.3\%.

CONCLUSION The local incidence of hydronephrosis in patients with severe uterovaginal or vault prolapse was $20.6 \%$ in our study. We established that $3.3 \%$ of women with severe uterovaginal or vault prolapse had mild renal impairment. Treatment by vaginal surgery for severe uterovaginal or vault prolapse appears to result in either complete resolution or improvement of hydronephrosis in the majority of patients.

Keywords: hydronephrosis, incidence, prolapse, uterovaginal, vault

\section{INTRODUCTION}

It is known that if left untreated, women with severe uterovaginal or vault prolapse may have hydronephrosis ${ }^{(1)}$ or renal impairment. ${ }^{(2)}$ The prevalence of hydronephrosis in patients undergoing treatment for pelvic organ prolapse has been reported to be in the range of $7.7 \%$ to $17 \% .^{(1,3,4)}$ It has been demonstrated that the prevalence of hydronephrosis significantly increases with the severity of prolapse. ${ }^{(4)}$ In this study, we aimed to: (a) evaluate the local incidences of hydronephrosis and renal impairment in the presence of severe uterovaginal or vault prolapse; and (b) determine whether treatment by surgery to correct the prolapse or the use of ring pessary resulted in the resolution of hydronephrosis in these patients.

\section{METHODS}

This was a retrospective case study of 121 patients who presented with severe uterovaginal or vault prolapse to the urogynaecology centre at KK Women's and Children's Hospital, Singapore, from March 2006 to December 2007. Clinical data were collected from the medical records of all patients. Patients who had known hydronephrosis due to other causes such as urinary tract obstruction secondary to abdominal or pelvic tumour, obstructive renal calculus, vesicoureteric reflux and ureteric strictures were excluded from the study. Ethical clearance was obtained for this study from the local central institutional review board.
All patients with grade 4 cystocoele and/or fourth degree uterovaginal prolapse or vault descent, and had undergone renal ultrasonography and renal function blood tests for the estimation of serum urea and creatinine, were included in the study. The severity of prolapse in the study population was assessed using the Baden-Walker Halfway System. ${ }^{(5)}$ All clinicians used the same classification system to assess the type and degree of prolapse in these patients during examination. Renal ultrasonography was performed by radiologists who were blinded to all clinical information. Patients diagnosed with hydronephrosis on imaging were evaluated using repeat renal ultrasonography after treatment (range 1-6 months) to determine the resolution of hydronephrosis. Resolution of hydronephrosis was defined as no residual hydronephrosis on renal ultrasonography 1-6 months after intervention. The extent of renal impairment was assessed based on evaluations of serum urea and creatinine levels.

Treatment for severe prolapse was mainly by surgery either vaginal hysterectomy coupled with pelvic floor repair and concomitant unilateral sacrospinous fixation, or vaginal hysterectomy with concomitant use of the Prolift mesh. All surgeries were performed via the vaginal route by two surgeons who were both consultant urogynaecologists at our centre. Some patients either defaulted follow-up or refused surgery, opting for silicone ring pessary instead.

All statistical analyses were performed using the Statistical Package for the Social Sciences for Windows version 16 (SPSS

${ }^{1}$ Department of Obstetrics and Gynaecology, ${ }^{2}$ Department of Urogynaecology, KK Women's and Children's Hospital, Singapore

Correspondence: Dr Wee Wei-Wei, Associate Consultant, Department of Obstetrics and Gynaecology, KK Women's and Children's Hospital, 100 Bukit Timah Road, Singapore 229899. wcube@hotmail.com 


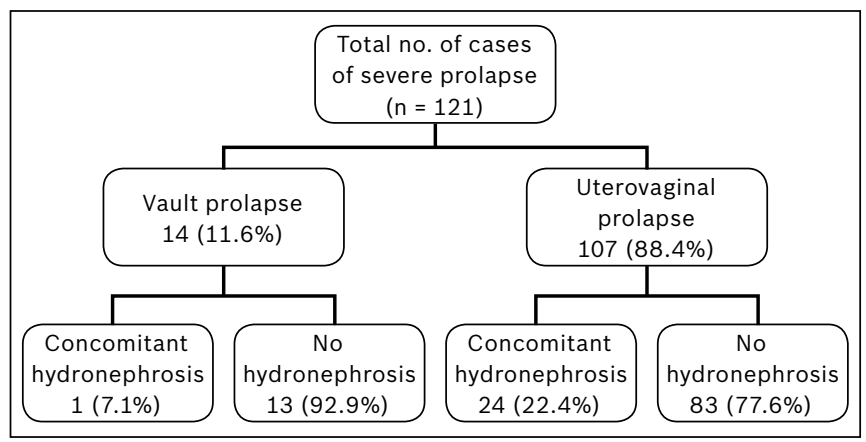

Fig. 1 Breakdown of patients with severe uterovaginal or vault prolapse.

Inc, Chicago, IL, USA) and OpenEpi version 2.2 (Open Source Epidemiologic Statistics for Public Health. Available at: www. OpenEpi.com). The chi-square test was used for bivariate analysis to test for statistical differences. A p-value $<0.05$ was considered statistically significant. Logistic regression was used for multivariate analysis.

\section{RESULTS}

Fig. 1 depicts the breakdown of patients with severe uterovaginal or vault prolapse in our study. Among the 121 patients with severe uterovaginal or vault prolapse, $14(11.6 \%)$ had vault prolapse and $107(88.4 \%)$ had uterovaginal prolapse. The mean age of the study population was 66.1 years. According to the Baden-Walker Halfway System, (5) $97.6 \%$ of the patients had concurrent grade 4 cystocoele and $59.7 \%$ had concurrent grade 4 rectocoele. All 107 patients with intact uterus had fourth degree cervical descent. Of the 14 patients who had previous hysterectomies, $85.7 \%$ of them had fourth degree vault descent.

The overall incidence of hydronephrosis among the patients in our study was $20.6 \%$ (25/121 patients). The incidence of hydronephrosis in patients with severe vault prolapse was $7.1 \%$ (1/14 patients), while that among patients with severe uterovaginal prolapse was $22.4 \%$ (24/107 patients). There was no statistically significant difference in the incidence of hydronephrosis between patients with severe uterovaginal prolapse and those with vault prolapse $(p=0.18)$. Among the 25 patients with hydronephrosis, $16(64.0 \%)$ had complete resolution of hydronephrosis after surgery or using ring pessary $(\mathrm{n}=1)$, while $5(20.0 \%)$ had residual but smaller degrees of hydronephrosis on follow-up ultrasonography (Table I). Four (16.0\%) patients defaulted and were lost to follow-up.

The incidence of renal impairment was 3.3\% (4/121 patients had procidentia). The serum urea (normal $<7.7 \mathrm{mmol} / \mathrm{L}$; range 10.3-18.9 mmol/L) and creatinine (normal < $141 \mu \mathrm{mol} / \mathrm{L}$; range $143-216 \mu \mathrm{mol} / \mathrm{L}$ ) levels were found to be elevated in the study population. All four patients with renal impairment had hydronephrosis in addition to fourth degree prolapse, indicating that patients with fourth degree prolapse in the presence of hydronephrosis had a $16 \%$ chance of having renal impairment in our cohort. Post-treatment improvement in serum creatinine levels was seen in all four patients with renal impairment.
Table I. Outcome of hydronephrosis in patients with severe uterovaginal or vault prolapse $(n=25)$.

\begin{tabular}{lc}
\hline Outcome/intervention & No. (\%) \\
\hline Fully resolved & $16(64)$ \\
Ring pessary & 1 \\
Surgery for uterovaginal prolapse & 15 \\
Partially resolved & $5(20)$ \\
Surgery for vault prolapse & 1 \\
Surgery for uterovaginal prolapse & 4 \\
Status unknown & $4(16)$ \\
Defaulted follow-up after surgery & 1 \\
Defaulted follow-up & 3 \\
\hline
\end{tabular}

\section{DISCUSSION}

Few studies have addressed hydronephrosis and renal impairment in the presence of severe uterovaginal or vault prolapse in the local population, and the outcome of treatment in these patients. Our study provides local data and results on such patients in Singapore. The local incidence of hydronephrosis in patients with severe uterovaginal or vault prolapse was $20.6 \%$ at our centre, which was slightly higher than that reported in other studies. ${ }^{(1,3,4)}$ We also established that $3.3 \%$ of women with severe uterovaginal or vault prolapse had mild renal impairment (serum creatinine level 143-216 $\mu \mathrm{mol} / \mathrm{L}$ ). This incidence of renal impairment increased to $16.0 \%$ among patients who had both fourth degree prolapse and concomitant hydronephrosis.

It is well established that untreated hydronephrosis may progress to irreversible renal damage. ${ }^{(2,6)}$ In this study, there was improvement in the serum creatinine levels of the four patients with renal impairment post procedure. This suggests that the resolution of hydronephrosis results in the favourable recovery of renal function. Our results also suggest that treating prolapse with vaginal surgery leads to the complete resolution or improvement of hydronephrosis in a majority of patients. In this study, $64 \%$ of patients showed complete resolution of hydronephrosis after surgical management. Unlike our findings, the incidence of resolution following management (95\%) was much higher in a prospective study by Hui et al. ${ }^{(4)}$ Our results indicate that it may be prudent to consider performing renal ultrasonography and renal function blood tests on patients with grade 4 uterovaginal or vault prolapse, with a view to offering them definitive treatment by surgery. Renal ultrasonography for all patients with grade 4 uterovaginal or vault prolapse is currently routine practice at our urogynaecology centre.

This study is not without its limitations. Firstly, this was a retrospective study with a small sample size of 121 patients. Collection of prospective data over a five-year period to increase the sample size would add value to the results of this study. Secondly, vaginal surgeries for our cohort were performed by two different surgeons; this may have contributed to confounding variables. Other than that, post-treatment renal ultrasonography should ideally be performed at a fixed interval of three months following surgical treatment for all patients in order to more accurately assess the resolution of hydronephrosis. However, 
this was not possible to enforce in our cohort, as some patients defaulted on their renal ultrasonography appointments and had to be rescheduled.

To conclude, the local incidence of hydronephrosis in patients with severe uterovaginal or vault prolapse was $20.6 \%$ in our study. $3.3 \%$ of women with severe uterovaginal or vault prolapse had mild renal impairment. In the majority of patients, treatment for severe uterovaginal or vault prolapse by vaginal surgery resulted in either complete resolution or improvement of hydronephrosis. Studies with longer follow-up periods and larger sample sizes are needed to further corroborate the results of this study.

\section{REFERENCES}

1. Beverly CM, Walters MD, Weber AM, Piedmonte MR, Ballard LA. Prevalence of hydronephrosis in patients undergoing surgery for pelvic organ prolapse. Obstet Gynecol 1997; 90:37-41.

2. Moore S, Bailey RR, Maling TM, Little PJ. Urinary tract obstruction and renal failure due to uterine prolapse. N Z Med J 1978; 87:429-31.

3. Gemer O, Bergman M, Segal S. Prevalence of hydronephrosis in patients with genital prolapse. Eur J Obstet Gynecol Reprod Biol 1999; 86:11-3.

4. Hui SY, Chan SC, Lam SY, Lau TK, Chung KH. A prospective study on the prevalence of hydronephrosis in women with pelvic organ prolapse and their outcomes after treatment. Int Urogynecol 2011; 22:1529-34.

5. Baden WF, Walker T. Fundamentals, symptoms, and classification. In: Baden WF, Walker T, eds. Surgical Repair of Vaginal Defects. Philadelphia: Lippincott, 1992: 9-23.

6. Churchill DN, Afridi S, Dow D, McManamon P. Uterine prolapse and renal dysfunction. J Urol 1980; 124:899-900.

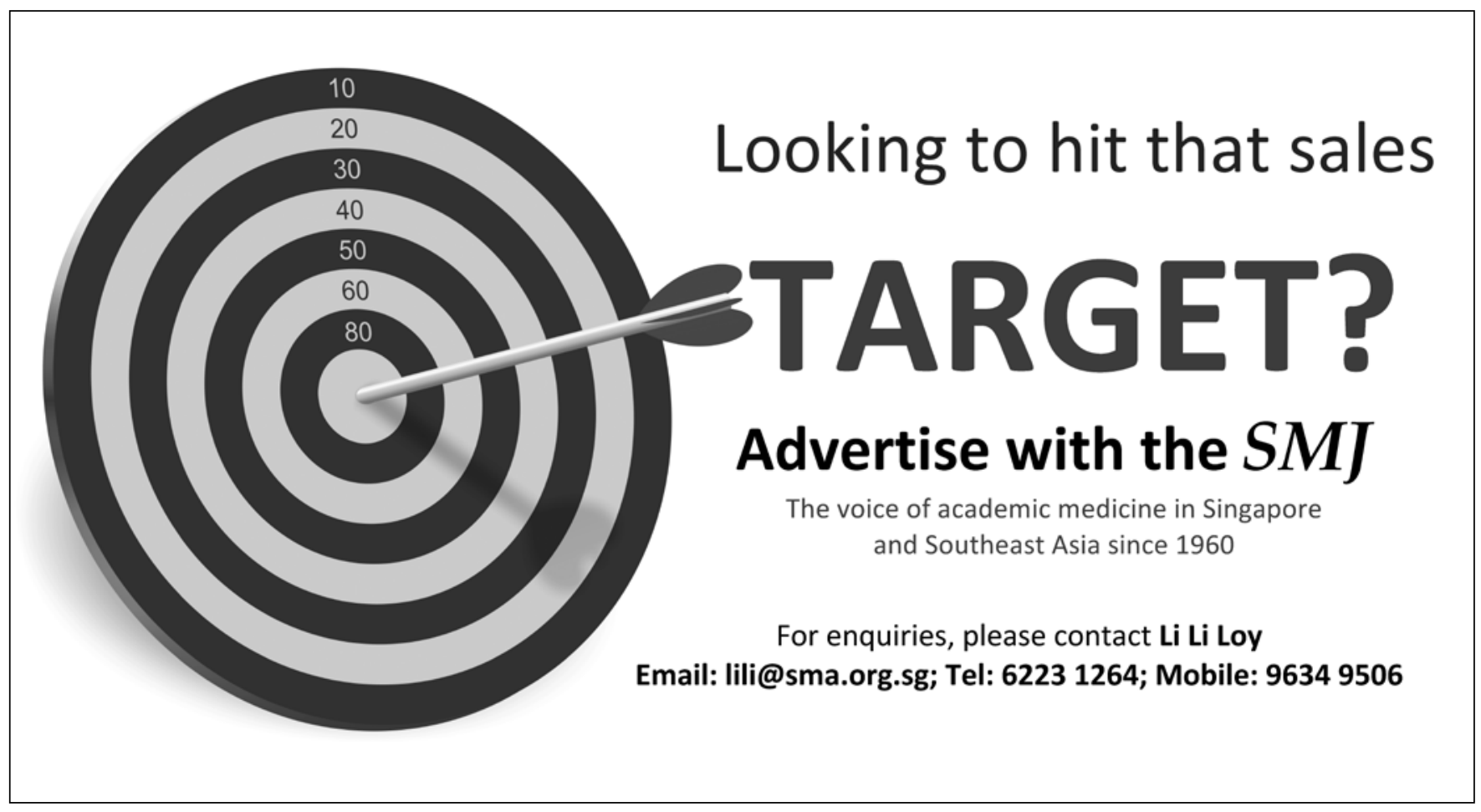

